\title{
A double-cell foam-filled composite block for efficient energy absorption under axial compression
}

\begin{abstract}
This paper describes an experimental evaluation of the crashworthiness characteristics of a novel design for cost-effective crashworthy composite glass fibre-reinforced plastic (GFRP) sandwich structures. All the samples are based on the concept of the "double-layered" foamfilled block, i.e. two foam-core sheets which are wrapped by reinforcement woven fabric, that acts as the reinforcement face and meanwhile ties the core layers and faces together, thus preventing catastrophic failure under axial loading conditions. The design, manufacturing and crush testing of rectangular blocks fabricated are described. Special attention is focused on the analysis of the mechanics of the block axial collapse, emphasizing on the mechanisms related to the crash energy absorption during the compression of the composite blocks with and without use of two types collapse trigger mechanism. Experimental results indicated high crushing force efficiency was achieved up to $80 \%$.
\end{abstract}

Keyword: Composite sandwich structure, Crashworthiness, Double-layered, Fibreglass fabric, Polyurethane foam, Trigger mechanism 